\title{
Influence of Enamel Conditioning on the Shear Bond Strength of Different Adhesives
}

\section{Einfluss der Schmelzkonditionierung auf die Scherkräfte unterschiedlicher Adhäsive}

\author{
Lorenz Brauchli ${ }^{*}$, Teodoro Muscillo ${ }^{1^{*}}$, Markus Steineck$^{1}$, Andrea Wichelhaus ${ }^{2}$
}

\begin{abstract}
Background and Objective: Phosphoric acid etching is the gold standard for enamel conditioning. However, it is possible that air abrasion or a combination of air abrasion and etching might result in enhanced adhesion. The aim of this study was to investigate the effect of different enamel conditioning methods on the bond strength of six adhesives.

Material and Methods: Three different enamel conditioning procedures (phosphoric acid etching, air abrasion, air abrasion + phosphoric acid etching) were evaluated for their influence on the shear bond strength of six different adhesives (Transbond ${ }^{\mathrm{TM}}$ XT, Cool-Bond ${ }^{\mathrm{TM}}$, Fuji Ortho LC, Ultra Band-Lok, Tetric ${ }^{\circledR}$ Flow, Light-Bond ${ }^{\mathrm{TM}}$ ). Each group consisted of 15 specimens. Shear forces were measured with a universal testing machine. The scores of the Adhesive Remnant Index (ARI) were also analyzed.

Results: There were no significant differences between phosphoric acid etching and air abrasion + phosphoric acid etching. Air abrasion as a single conditioning technique led to significantly lower shear forces. The ARI scores did not correlate with the shear strengths measured. There were greater variations in shear forces for the different adhesives than for the conditioning techniques.

Conclusion: The highest shear forces were found for the conventional composites Transbond ${ }^{\mathrm{TM}} \mathrm{XT}$ and CoolBond $^{\mathrm{TM}}$ in combination with conventional etching. Air abrasion alone and in combination with phosphoric acid
\end{abstract}

\footnotetext{
Clinic of Orthodontics and Pedodontics, Dental School, University of Basel, Switzerland,

2 Department of Orthodontics, Ludwig Maximilian University, Munich, Germany

*These authors contributed equally to this research.
}

Received: August 18, 2010; accepted: September 16, 2010

\begin{abstract}
Zusammenfassung
Hintergrund und Ziel: Phosphorsäure-Ätzung ist der Goldstandard in der Schmelzkonditionierung. Es ist jedoch denkbar, dass Sandstrahlung oder eine Kombination von Sandstrahlung und Ätzung zu einer erhöhten Schmelzadhäsion führen könnten. Ziel der vorliegenden Studie war die Untersuchung des Einflusses unterschiedlicher Schmelzkonditionierungsmethoden auf die Haftkräfte von sechs Adhäsivstoffen.
\end{abstract}

Material und Methodik: Drei unterschiedliche Schmelzkonditionierungen (Phosphorsäure, Sandstrahlung, Sandstrahlung und Phosphorsäure) wurden bezüglich ihres Einflusses auf die Scherkräfte von sechs unterschiedlichen Adhäsiven (Transbond $^{\mathrm{TM}}$ XT, Cool-Bond ${ }^{\mathrm{TM}}$, Fuji Ortho LC, Ultra Band-Lok, Tetric ${ }^{\circledR}$ Flow, Light-Bond ${ }^{\mathrm{TM}}$ ) untersucht. Jede Gruppe bestand aus 15 Proben. Die Scherkräfte wurden mittels einer Universalprüfmaschine gemessen. Zusätzlich wurde ein Adhäsiv-Rest-Index (ARI) bestimmt.

Ergebnisse: Insgesamt gab es keine signifikanten Unterschiede zwischen den Gruppen Phosphorsäure-Ätzung und Sandstrahlung + Phosphorsäure-Ätzung. Sandstrahlung als alleinige Konditionierungsmethode führte zu signifikant niedrigeren Scherkräften. Die ARI-Werte korrelierten nicht mit den gemessenen Scherkräften. Die Variation der Scherkräfte war für unterschiedliche Adhäsivsysteme gröBer als für unterschiedliche Konditionierungen.

Schlussfolgerung: Die höchsten Scherkräfte wurden für die konventionellen Komposite Transbond ${ }^{\mathrm{TM}}$ XT und CoolBond $^{\mathrm{TM}}$ in Kombination mit konventioneller Ätzung gefunden. Sandstrahlung in Kombination mit Phosphorsäure-

J Orofac Orthop 2010;71:411-20

DOI $10.1007 / \mathrm{s} 00056-010-1036-2$ 
etching showed no advantages compared with phosphoric acid etching alone and, therefore, cannot be recommended.

Key Words: Shear force - Bovine enamel · Etching - Air abrasion $\cdot$ In-vitro study

\section{Introduction}

The effectiveness of the bonding at the enamel-adhesive interface depends on two variables: the conditioning of the enamel and the adhesive. Ever since Buonocore [6] described the etching of enamel with $85 \%$ phosphoric acid, this procedure, albeit with less concentrated acid, has become the standard for enamel conditioning. It modifies the enamel surface to create a typical etching pattern $[1,35]$. The acid concentrations for etching reported in the literature range from $2 \%$ [9] to $85 \%$ [6] with application times of between 15 and 120 seconds [25]. Most authors describe the application of a $35-40 \%$ solution and a duration of etching of 15-60 seconds $[14,22]$. It is estimated that etching can lead to an enamel loss of between 10 and 30 microns [36], which might be partly reversible if moderate etching times and acid concentrations are used [35].

In contrast to etching, air abrasion does not chemically modify the enamel surface. Fine particles, usually aluminum oxides in sizes of 25 to 50 microns, are accelerated to a velocity of up to $20 \mathrm{~m} / \mathrm{s}$. The impact of these aluminum particles makes pyramid-shaped indentations and thus roughens the enamel surface. The procedure leads to a significant loss of surface enamel [21]. The effect of air abrasion as discussed in the literature is controversial [8, 15, 21, 35]. Most authors found significantly lower shear bond strengths with air abrasion. Jost-Brinkmann et al. [15] however found shear forces equal to conventionally etched enamel. The differing results may be due to several factors such as varying particle size and material, particle velocity, distance to the tooth surface and duration of abrasion.

In conclusion, the combination of air abrasion and conventional etching has not been well documented in the literature and it seems that combining the two techniques does not enhance bond strength above the level achieved by etching alone [8].

One of the main factors relevant to shear strengths would appear to be the choice of adhesive. Higher bonding forces were reported for composites than for resin-modified glass ionomer cements (RMGIC) [1, 8, 26, 29]. But significant differences were also found between adhesives of the same type $[12,15,35]$. Furthermore, the supposed disadvantage of RMGIC in terms of adhesive strength identified in some in-vitro studies might be compensated for by their higher tolerance to humidity $[7,17,19,28]$.
Ätzung zeigte keinen Vorteil gegenüber alleiniger Phosphorsäure-Ätzung und kann deshalb nicht empfohlen werden.

Schlüsselwörter: Scherkräfte · Boviner Schmelz • Ätzung · Sandstrahlung $\cdot$ In-vitro-Studie

\section{Einleitung}

Die Hafteigenschaften der Schmelz-Adhäsiv-Grenzfläche sind abhängig von zwei Variablen: der Konditionierung des Schmelzes und des verwendeten Adhäsivstoffes. Seit Buonocore [6] die Ätzung von Schmelz mit 85\%iger Phosphorsäure beschrieben hatte, wurde dieses Verfahren, wenn auch mit weniger konzentrierter Säure, zum Standard der Schmelzkonditionierung. Das Ätzverfahren führt zu einer Modifikation der Schmelzoberfläche mit typischem Ätzmuster $[1,35]$. In der Literatur wurden unterschiedliche Konzentrationen der Phosphorsäure zwischen 2\% [9] und 85\% [6] und unterschiedliche Applikationszeiten zwischen 15 und 120 Sekunden [25] gefunden. Die meisten Autoren beschreiben eine Anwendung von 35-40\%iger Phosphorsäure bei einer Einwirkungszeit zwischen 15 und 60 Sekunden [14, 22]. Der Schmelzverlust wurde auf ca. 10-30 $\mu \mathrm{m}$ [36] geschätzt, wobei eine partielle Reversibilität bei gemäßigten Ätzzeiten und Säurekonzentrationen diskutiert wurde [35].

Im Gegensatz zur Ätzung führt die Sandstrahlung nicht zu einer chemischen Modifizierung der Schmelzoberfläche. Kleine Partikel, zumeist aus Aluminiumoxid in Größen von $25-50 \mu \mathrm{m}$, werden auf eine Beschleunigung von bis zu $20 \mathrm{~m} / \mathrm{s}$ gebracht. Die Aluminiumpartikel hinterlassen auf der Schmelzoberfläche pyramidenförmige Einschusskrater und führen dadurch zu einer Aufrauung. Das Verfahren geht mit einem signifikanten Verlust von oberflächlichem Schmelz einher [21]. Der Einfluss der Sandstrahlung wurde in der Literatur unterschiedlich diskutiert $[8,15,21,35]$. Die meisten Autoren fanden signifikant niedrigere Haftkräfte mit Sandstrahlung. Jost-Brinkmann et al. [15] hingegen fanden ähnliche Scherkräfte wie bei konventionell geätztem Schmelz. Verschiedene Faktoren müssen für die divergierenden Resultate diskutiert werden. Die Sandstrahlung kann mit unterschiedlichen Partikelgrößen und Materialien, Beschleunigung der Partikel, Distanzen zur Zahnoberfläche und unterschiedlicher Abrasionsdauer durchgeführt werden.

Eine Kombination von Sandstrahlung und konventioneller Ätzung könnte zu einer Erhöhung der Haftwerte führen. Allerdings ist dieser Effekt in der Literatur nicht gut belegt. Vielmehr scheint die kombinierte Anwendung beider Techniken die Haftfestigkeit nicht über das Niveau der Konditionierung nach alleinigem Ätzvorgang anzuheben [8].

Einer der wichtigsten Faktoren mit Relevanz für die Scherkräfte scheint die Wahl des Adhäsivs zu sein. Für Kom- 
The aim of this study was to evaluate whether air abrasion, or a combination of air abrasion and conventional etching produce superior bond strengths in comparison to etching alone and whether different types of adhesive, such as composites or resin-modified glass ionomer cements (RMGIC), behave differently when combined with the conditioning techniques under investigation.

\section{Materials and Methods}

270 bovine incisors were extracted and stored in $37{ }^{\circ} \mathrm{C}$ deionized water after removal of the pulp. They were embedded in polymer blocks (Technovit 4071 Kaltpolymerisat; Heraeus-Kulzer, Wehrheim, Germany) prior to further treatment. The anterior surface of the polymer blocks was trimmed parallel to the labial surface of the bovine incisors.

Six different adhesives were tested in combination with three conditioning techniques (Table 1). Four of the adhesives were composites: Transbond ${ }^{\mathrm{TM}}$ XT (3M Unitek, Monrovia, CA, USA), Cool-Bond ${ }^{\mathrm{TM}}$ (Dentronix ${ }^{\circledR}$, Cuyahoga Falls, $\mathrm{OH}$, USA), Light-Bond ${ }^{\mathrm{TM}}$ (Reliance Orthodontic Products, Itasca, IL, USA), Tetric ${ }^{\circledR}$ flow (Ivoclar Vivadent, Schaan, Liechtenstein). The remaining adhesives were RMGIC: GC Fuji Ortho LC (GC Europe, Leuven, Belgium), and Ultra Band-Lok (Reliance Orthodontic Products, Itasca, IL, USA).

For each adhesive 45 samples were produced, resulting in a total of 15 samples per adhesive and conditioning technique. The enamel surface of all specimens was cleaned with a rotating brush and pumice, rinsed and dried with compressed air.

In the control group, 35\% phosphoric acid (Unitek ${ }^{\mathrm{TM}}$ Etching Gel; 3M Unitek, Monrovia, CA, USA) was applied to the teeth for 30 seconds, then the enamel was thoroughly rinsed with water and dried with air before bonding. In the air-abrasion group, the enamel was treated with an air-abrasion device (Air Abrasion; KaVo Dental AG, Brugg, Switzerland) using 50 micron aluminum oxide particles from a distance of $2 \mathrm{~mm}$ for 2 seconds. The specimens were rinsed with water and dried with air. The third group was treated with a combination of conditioning and air abrasion. Air abrasion and etching were carried out as described above, whereby air abrasion preceded etching.

Instead of using brackets, transparent polymer cylinders with a diameter of $6 \mathrm{~mm}$ were used as shear bodies (Karl Haug Kunststoffverarbeitung, Basel, Switzerland). The bonding surfaces of the polymethylmethacrylate (PMMA) cylinders were conditioned with the Rocatec system using aluminum oxide silica particles (Rocatec; 3M Espe, St. Paul, MN, USA).

Priming was carried out according to the instructions from the manufacturers. Light curing was applied for $20 \mathrm{sec}-$ onds with a Bluephase C8 (Ivoclar Vivadent, Schaan, Liechtenstein) which has a range of 430-490 nm and an intensity of $800 \mathrm{~mW} / \mathrm{cm}^{2}$. After bonding of the bracket substitutes, the specimens were again stored in $37^{\circ} \mathrm{C}$ deionized water. posite wurden höhere Haftkräfte angegeben als für kompositmodifizierte Glasionomerzemente (RMGIC) [1, 8, 26, 29]. Allerdings wurden auch signifikante Unterschiede zwischen den Adhäsiven desselben Typus gefunden [12, 15, 35]. Zudem könnte der Nachteil von RMGIC im Hinblick auf die absoluten Haftwerte durch eine höhere Feuchtigkeitstoleranz [7, 17, 19, 28] ausgeglichen werden.

Das Ziel der vorliegenden Studie war die Evaluation der Frage, ob die Techniken Sandstrahlung oder Sandstrahlung in Kombination mit konventioneller Ätzung höhere Haftfestigkeiten erzielen als die alleinige konventionelle Ätzung. Zudem sollten die Hafteigenschaften unterschiedlicher Adhäsivsysteme wie konventionelle Komposite oder kompositmodifizierte Glasionomerzemente (RMGIC) in Kombination mit den erwähnten Konditionierungstechniken untersucht werden.

\section{Material und Methodik}

270 Rinderinzisivi wurden extrahiert und in $37^{\circ} \mathrm{C}$ warmem, deionisiertem Wasser nach Entfernung der Pulpa aufbewahrt. Vor der weiteren Behandlung wurden die Zähne in Polymerblöcke (Technovit 4071 Kaltpolymerisat; HeraeusKulzer, Wehrheim, Deutschland) eingebettet. Die vordere Oberfläche der Polymerblöcke wurde parallel zu den Labialflächen der Rinderzähne getrimmt.

Sechs unterschiedliche Adhäsive wurden in Kombination mit drei Konditionierungstechniken untersucht (Tabelle 1). Vier der Adhäsivsysteme waren Komposite: Transbond ${ }^{\mathrm{TM}}$ XT (3M Unitek, Monrovia, CA, USA), Cool-Bond ${ }^{\mathrm{TM}}$ (Dentronix $^{\circledR}$, Cuyahoga Falls, OH, USA), Light Bond ${ }^{\mathrm{TM}}$ (Reliance Orthodontic Products, Itasca, IL, USA), Tetric ${ }^{\circledR}$ flow (Ivoclar Vivadent, Schaan, Liechtenstein). Die übrigen Adhäsive waren kompositmodifizierte Glasionomerzemente: GC Fuji Ortho LC (GC Europe, Leuven, Belgien) und Ultra Band-Lok (Reliance Orthodontic Products, Itasca, IL, USA).

Für jede Adhäsivgruppe wurden 45 Proben hergestellt, was in 15 Proben pro Adhäsiv und Konditionierungstechnik resultierte. Die Schmelzoberflächen aller Proben wurden mit einer rotierenden Bürste und Bimsstein gereinigt, mit Wasser gespült und mit komprimierter Luft getrocknet. Für die Kontrollgruppe wurde $35 \%$ ige Phosphorsäure (Unitek ${ }^{\mathrm{TM}}$ Etching Gel; 3M Unitek, Monrovia, CA, USA) für 30 Sekunden auf die Zähne appliziert. Anschließend wurde der Schmelz gründlich mit Wasser gespült und mit Luft getrocknet.

Für die Sandstrahlgruppe wurde der Schmelz mit einem Sandstrahlgerät (Air Abrasion; KaVo Dental AG, Brugg, Schweiz) unter Verwendung von $50 \mu \mathrm{m}$ Aluminium-OxidPartikeln und aus einer Distanz von $2 \mathrm{~mm}$ für 2 Sekunden behandelt. Die Proben wurden mit Wasser gespült und mit Luft getrocknet. Die dritte Gruppe wurde mit einer Kombination von Sandstrahlung und Ätzung vorbehandelt. Beide Konditionierungstechniken wurden entsprechend der oben beschriebenen Vorgehensweise durchgeführt, wobei die Ätzung der Sandstrahlung folgte. 


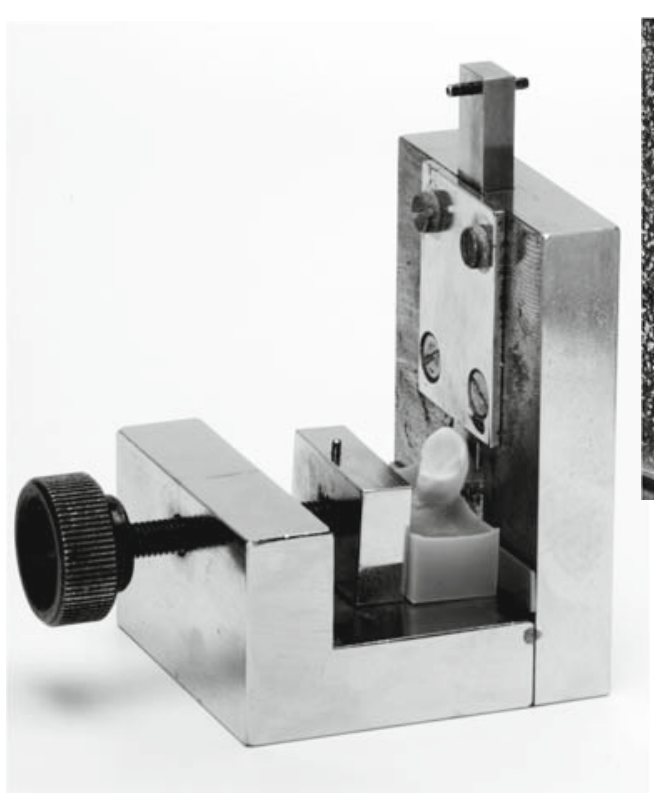

For shear testing, the specimens were inserted into a shear force jig (Figure 1) and measured with a universal testing machine (Instron 4444; Instron Corp., Wilmington, DE, USA) with a crosshead speed of $0.1 \mathrm{~mm} / \mathrm{s}$. The distance of the labial surface of the tooth to the shear blade of the jig was set to $1.5 \mathrm{~mm}$. The data was stored on a PC using the software Origin 6.1 (OriginLab, Northampton, MA, USA).

In addition to shear force testing, an Adhesive Remnant Index (ARI) was recorded under 10× optical magnification: ARI 1) $<10 \%$ adhesive left on the bracket, ARI 2) cohesive fracture, or equal distribution of composite left on the bracket and tooth surface, ARI 3) $<10 \%$ adhesive left on the enamel.

Descriptive statistics with mean, median, standard deviation (SD) and standard error of the mean (SEM) were calculated. Statistically the data sets were tested for normal distribution (Kolmogorov-Smirnov) and evaluated with a two-factor ANOVA using a Tukey post-hoc test. The two factors of interest being the adhesive and the conditioning technique in regard to shear forces. The level of significance was set at $\mathrm{p} \leq 0.05$. A nonparametric Kruskal-Wallis test was calculated in addition to the ANOVA because three of the 18 groups did not show normal distribution.

\section{Results}

The results were evaluated according to the type of adhesive as well as the conditioning technique and are summarized in Tables 1 to 3 and Figure 2.

Three data sets did not pass the Kolmogorov-Smirnov test for normal distribution: Cool-Bond ${ }^{\mathrm{TM}}$ (air abrasion, air abrasion in combination with etching), Transbond ${ }^{\mathrm{TM}} \mathrm{XT}$ (air abrasion in combination with etching).

Overall Transbond ${ }^{\mathrm{TM}} \mathrm{XT}$ attained the highest shear values $(\bar{x}=13.3 \mathrm{MPa})$, followed by Cool-Bond ${ }^{\mathrm{TM}}(\bar{x}=11.7$
Anstelle der Verwendung von Brackets wurden Polymerzylinder (Karl Haug Kunststoffverarbeitung, Basel, Schweiz) mit einem Durchmesser von $6 \mathrm{~mm}$ als Scherkörper verwendet. Die Bondingoberfläche der Polymerzylinder wurde mit dem Rocatec-System behandelt, wodurch Aluminiumoxid-Silizium-Partikel aufgebracht wurden (Rocatec; 3M Espe, St. Paul, MN, USA).

Die Klebung erfolgte entsprechend den Herstellerangaben. Die Lichthärtung wurde für 20 Sekunden mit einer Bluephase C8 (Ivoclar Vivadent, Schaan, Liechtenstein) mit einer Wellenlänge von 430-490 nm und einer Intensität von 800 $\mathrm{mW} / \mathrm{cm} 2$ durchgeführt. Nach dem Bonding der Bracket-Analoga wurden die Proben erneut in $37^{\circ} \mathrm{C}$ warmem, deionisiertem Wasser gelagert.

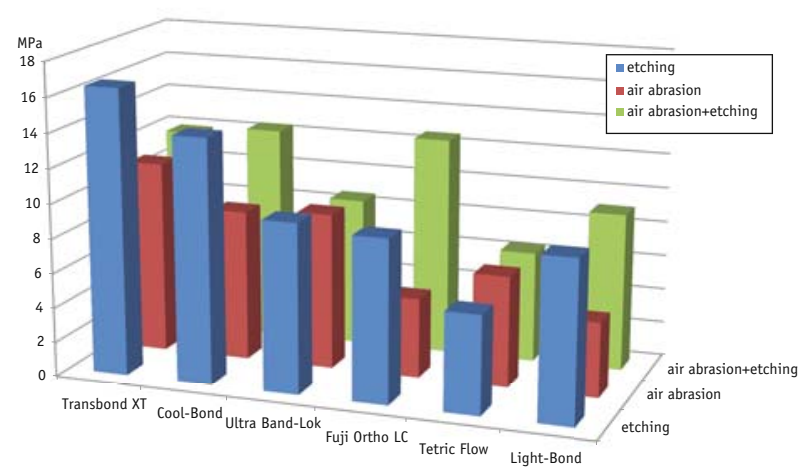

Figure 2. Shear forces in MPa of all adhesives and conditioning methods. Air abrasion without etching led to significantly lower shear forces.

Abbildung 2. Scherkräfte (Angabe in MPa) aller Adhäsive in Kombination mit drei Konditionierungsmethoden. Alleinige Sandstrahlung führte zu signifikant niedrigeren Scherkräften. 
Table 1. Number of samples classified according to the adhesive remnant index. ARI 1) $<10 \%$ adhesive left on the bracket, ARI 2) cohesive fracture, or equal distribution of adhesive left on the bracket and tooth surface, ARI 3) $<10 \%$ adhesive left on the enamel.

Tabelle 1. Einteilung der Proben nach dem Adhäsiv-Rest-Index (ARI). ARI 1: $<10 \%$ Adhäsiv auf dem Bracket verbleibend. ARI 2: Kohäsive Fraktur mit gleichmäßiger Verteilung des Adhäsives. ARI 3: $<10 \%$ des Adhäsiv auf dem Schmelz verbleibend.

\begin{tabular}{|c|c|c|c|c|}
\hline & $\begin{array}{l}\text { Conditioning } \\
\text { method }\end{array}$ & $\begin{array}{l}\text { ARI } 1 \\
\text { bracket }\end{array}$ & $\begin{array}{l}\text { ARI } 2 \\
\text { adhesive }\end{array}$ & $\begin{array}{l}\text { ARI } 3 \\
\text { enamel }\end{array}$ \\
\hline Transbond $^{\mathrm{TM}} \mathbf{X T}$ & etching & 1 & 3 & 11 \\
\hline 3M Unitek, & air abrasion & 4 & 7 & 4 \\
\hline Monrovia. CA, USA & air abrasion + etching & 5 & 6 & 4 \\
\hline Cool-Bond $^{\mathrm{TM}}$ & etching & 1 & 4 & 10 \\
\hline Dentronix ${ }^{\mathrm{TM}}$ & air abrasion & 7 & 7 & 1 \\
\hline $\begin{array}{l}\text { Cuyahoga Falls, } \mathrm{OH} \text {, } \\
\text { USA }\end{array}$ & air abrasion + etching & 6 & 6 & 3 \\
\hline Ultra Band-Lok & etching & 2 & 10 & 3 \\
\hline Reliance Ortho. & air abrasion & 4 & 3 & 8 \\
\hline Prod., Itasca, IL, USA & air abrasion + etching & 1 & 10 & 4 \\
\hline GC Fuji Ortho LC & etching & 4 & 4 & 7 \\
\hline GC Europe N.V, & air abrasion & 10 & 3 & 2 \\
\hline Leuven, Belgium & air abrasion + etching & $\lg 2$ & 3 & 10 \\
\hline Tetric ${ }^{\circledR}$ Flow & etching & 8 & 5 & 2 \\
\hline Ivoclar Vivadent, & air abrasion & 10 & 3 & 2 \\
\hline Schaan, Liechtenstein & air abrasion + etching & $\lg 2$ & 3 & 10 \\
\hline Light-Bond ${ }^{\circledR}$ & etching & 1 & 6 & 8 \\
\hline Reliance Ortho. & air abrasion & 10 & 3 & 2 \\
\hline Prod., Itasca, IL, USA & air abrasion + etching & 2 & 6 & 7 \\
\hline
\end{tabular}

MPa), Ultra Band-Lok $(\bar{x}=9.1 \mathrm{MPa})$, GC Fuji Ortho LC $(\bar{x}=8.9 \mathrm{MPa})$, Light-Bond ${ }^{\mathrm{TM}}(\bar{x}=7.5 \mathrm{MPa})$ and Tetric ${ }^{\circledR}$ Flow $(\bar{x}=6.2 \mathrm{MPa})$. No significant difference was found between Transbond ${ }^{\mathrm{TM}} \mathrm{XT}$ and Cool-Bond ${ }^{\mathrm{TM}}$ but Transbond ${ }^{\mathrm{TM}}$ XT differed in fact from all the other adhesives. The shear strengths recorded for the two RMGICs were medium-sized, significantly lower than Transbond ${ }^{\mathrm{TM}} \mathrm{XT}$, but equal to CoolBond $^{\mathrm{TM}}$ and significantly higher than Tetric ${ }^{\circledR}$ Flow and LightBond $^{\mathrm{TM}}$.

Considering the different enamel conditioning techniques, the highest shear forces for conventional etching $(\bar{x}=16.5 \mathrm{MPa})$ and air abrasion $(\bar{x}=11.1 \mathrm{MPa})$ were found for Transbond ${ }^{\mathrm{TM}}$ XT. For air abrasion combined with etching, the composite-modified glass ionomer cement Fuji Ortho LC yielded the highest bond strengths $(\bar{x}=12.6 \mathrm{MPa})$. The lowest shear forces for conventional etching $(\bar{x}=5.7 \mathrm{MPa})$ and air abrasion combined with etching $(\bar{x}=6.4 \mathrm{MPa})$ were found for Tetric $^{\circledR}$ Flow. For air abrasion alone, Light-Bond ${ }^{\mathrm{TM}}$ showed the lowest adhesive strength $(\bar{x}=4.3 \mathrm{MPa})$.

Significant differences between the different conditioning techniques were found regardless of the type of adhesive (Table 3). The conventional etching procedure resulted in the highest mean bonding forces $(\bar{x}=10.8 \mathrm{MPa})$, followed by the combination of air abrasion and etching $(\bar{x}=9.6$
Zur Schertestung wurden die Proben in einen Abschermechanismus eingebracht und mittels einer Universalprüfmaschine (Instron 4444; Instron Corp., Wilmington, DE, USA) bei einer Abschergeschwindigkeit von $0,1 \mathrm{~mm} / \mathrm{s}$ und einer Distanz des Abscherstempels zu der labialen Zahnoberfläche von 1,5 mm getestet (Abbildung 1). Die Daten wurden über einen PC mittels Origin 6.1 (OriginLab, Northampton, MA, USA) aufgezeichnet.

Zusätzlich wurde ein „Adhesive Remnant Index“ (ARI) unter zehnfacher optischer Vergrößerung aufgenommen: ARI 1) < 10\% Adhäsiv auf dem Bracket-Analogon verbleibend, ARI 2) Kohäsive Fraktur mit ähnlicher Verteilung der Kompositreste auf dem Bracket und der Zahnoberfläche, ARI 3) $<10 \%$ Adhäsiv auf dem Schmelz verbleibend.

Eine deskriptive Statistik mit Mittelwerten, Median, Standardabweichung (SD) und Standardmittelfehler (SEM) wurde berechnet. Der Datensatz wurde auf Normalverteilung mittels Kolmogorov-Smirnov-Test geprüft und mit einer 2-Faktor-ANOVA und einem Post-hoc-Tukey-Test ausgewertet. Die beiden Faktoren der Analyse waren das verwendete Adhäsiv und die Konditionierungsmethode im Vergleich zu den Scherkräften. Das Signifikanzniveau wurde auf $\mathrm{p} \leq 0,05$ festgelegt. Da drei der Gruppen keine Normalverteilung erreichten, wurde zusätzlich ein Kruskal-Wallis-Test durchgeführt.

\section{Ergebnisse}

Die Resultate wurden nach Adhäsivtypen und Konditionierungstechnik ausgewertet und in den Tabellen 1 bis 3 und Abbildung 2 zusammengefasst.

Drei Datensätze erreichten keine Normalverteilung im Kolmogorov-Smirnov-Test: Cool-Bond ${ }^{\mathrm{TM}}$ (Sandstrahlung, Sandstrahlung in Kombination mit Ätzung), Transbond ${ }^{\mathrm{TM}}$ XT (Sandstrahlung in Kombination mit Ätzung).

Insgesamt erreichte Transbond ${ }^{\mathrm{TM}} \mathrm{XT}$ die höchsten Scherkräfte $(\bar{x}=13,3 \mathrm{MPa})$, gefolgt von Cool-Bond ${ }^{\mathrm{TM}}(\bar{x}=11,7$ MPa), Ultra Band-Lok ( $\bar{x}=9,1 \mathrm{MPa})$, GC Fuji Ortho LC $(\bar{x}=8,9 \mathrm{MPa})$, Light-Bond ${ }^{\mathrm{TM}}(\bar{x}=7,5 \mathrm{MPa})$ und Tetric ${ }^{\circledR}$ Flow $(\bar{x}=6,2 \mathrm{MPa})$. Der Unterschied zwischen Transbond ${ }^{\mathrm{TM}}$ XT und Cool-Bond ${ }^{\mathrm{TM}}$ war nicht signifikant, Transbond ${ }^{\mathrm{TM}}$ XT unterschied sich jedoch von allen anderen Adhäsiven. Beide kompositmodifizierten Glasionomerzemente zeigten mittlere Scherkräfte. Diese waren signifikant niedriger als die Scherkräfte von Transbond ${ }^{\mathrm{TM}}$ XT, entsprachen den Kräften von Cool-Bond ${ }^{\mathrm{TM}}$ und lagen signifikant über den Kräften von Tetric $^{\circledR}$ Flow und Light-Bond ${ }^{\mathrm{TM}}$.

Betrachtet man die unterschiedlichen Schmelzkonditionierungstechniken, so zeigte Transbond ${ }^{\mathrm{TM}} \mathrm{XT}$ die höchsten Scherkräfte bei der konventionellen Ätzung $(\bar{x}=16,5 \mathrm{MPa})$ und Sandstrahlung ( $\bar{x}=11,1 \mathrm{MPa})$. Für Sandstrahlung in Kombination mit einer Ätzung wies der kompositmodifizierte Glasionomerzement Fuji Ortho LC die höchsten Haftwerte auf $(\bar{x}=12,6 \mathrm{MPa})$. Die niedrigsten Haftwerte wurden für Tetric $^{\circledR}$ Flow in Kombination mit konventioneller Ätzung 
Table 2. Shear forces measured for the different adhesives and conditioning methods, including mean, standard deviation (SD), standard error of mean (SEM) and median. Normal distribution was calculated according to Kolmogorov-Smirnov. The column "significance" shows significant differences to other groups within one conditioning method and the ranking (Kruskal-Wallis) gives an overall classification.

Tabelle 2. Scherkräfte der verschiedenen Adhäsive und Konditionierungsmethoden mit Mittelwerten, Standardabweichungen (SD), Standardmittelwertfehler (SEM) und Median. Die Normalverteilung wurde nach Kolmogorov-Smirnov getestet. Die Spalte "Significance“ weist signifikante Unterschiede zwischen den Gruppen innerhalb einer Konditionierungsmethode auf und „Ranking“ (Kruskal-Wallis) spiegelt die Klassifikation über alle Gruppen wider.

\begin{tabular}{|c|c|c|c|c|c|c|c|c|c|}
\hline Conditioning & $\begin{array}{l}\text { Goup } \\
\text { number }\end{array}$ & Adhesive & $\begin{array}{l}\text { Mean } \\
{[\mathrm{MPa}]}\end{array}$ & SD & SEM & $\begin{array}{l}\text { Median } \\
{[\mathrm{MPa}]}\end{array}$ & $\begin{array}{l}\text { Kolmogorov- } \\
\text { Smirnov, } \\
\text { p value }\end{array}$ & $\begin{array}{l}\text { Significance } \\
\text { (ANOVA, } \\
\mathbf{p} \leq 0.05 \text { ) }\end{array}$ & $\begin{array}{l}\text { Ranking } \\
\text { (Kruskal- } \\
\text { Wallis) }\end{array}$ \\
\hline \multirow[t]{6}{*}{ Etching } & 1 & Transbond $^{\mathrm{TM}} \mathrm{XT}$ & 16.5 & 9.9 & 2.6 & 13.4 & $>0.1$ & $3,4,5,6$ & 2 \\
\hline & 2 & Cool-Bond $^{\mathrm{TM}}$ & 14.1 & 3.8 & 1 & 14.7 & $>0.1$ & $3,4,5,6$ & 1 \\
\hline & 3 & Ultra Band-Lok & 9.8 & 2.5 & 0.65 & 9.9 & $>0.1$ & 1,2 & 5 \\
\hline & 4 & Fuji Ortho LC & 9.4 & 4.4 & 1.1 & 4.6 & $>0.1$ & 1,2 & 12 \\
\hline & 5 & Tetric ${ }^{\circledR}$ Flow & 5.7 & 2.6 & 0.7 & 5.3 & $>0.1$ & 1,2 & 16 \\
\hline & 6 & Light-Bond ${ }^{\mathrm{TM}}$ & 9.3 & 2.9 & 0.8 & 9.9 & $>0.1$ & 1,2 & 6 \\
\hline \multirow[t]{6}{*}{ Air abrasion } & 7 & Transbond $^{\mathrm{TM}} \mathrm{XT}$ & 11.1 & 4.8 & 1.3 & 12.5 & $>0.1$ & $10,11,12$ & 4 \\
\hline & 8 & Cool-Bond $^{\mathrm{TM}}$ & 8.7 & 5.2 & 1.3 & 7.6 & 0.016 & $10,11,12$ & 13 \\
\hline & 9 & Ultra Band-Lok & 9 & 3.9 & 1 & 9.2 & $>0.1$ & $10,11,12$ & 10 \\
\hline & 10 & Fuji Ortho LC & 4.6 & 4 & 1 & 4 & $>0.1$ & $7,8,9$ & 17 \\
\hline & 11 & Tetric $^{\circledR}$ Flow & 6.4 & 2.5 & 0.7 & 6.4 & $>0.1$ & $7,8,9$ & 15 \\
\hline & 12 & Light-Bond ${ }^{\mathrm{TM}}$ & 4.3 & 2.2 & 0.6 & 3.8 & $>0.1$ & $7,8,9$ & 18 \\
\hline \multirow{6}{*}{$\begin{array}{l}\text { Air abrasion } \\
\text { + etching }\end{array}$} & 13 & Transbond $^{\mathrm{TM}} \mathrm{XT}$ & 11.9 & 9.9 & 2.6 & 8.3 & $<0.001$ & & 9 \\
\hline & 14 & Cool-Bond $^{\mathrm{TM}}$ & 12.4 & 10.7 & 2.7 & 9.1 & $<0.001$ & & 7 \\
\hline & 15 & Ultra Band-Lok & 8.6 & 1.7 & 0.4 & 9 & $>0.1$ & & 11 \\
\hline & 16 & Fuji Ortho LC & 12.6 & 5.9 & 1.5 & 12.3 & $>0.1$ & 17 & 3 \\
\hline & 17 & Tetric $^{\circledR}$ Flow & 6.4 & 3 & 0.8 & 6.6 & $>0.1$ & 16 & 14 \\
\hline & 18 & Light-Bond ${ }^{\mathrm{TM}}$ & 9.1 & 2.2 & 0.6 & 3.8 & $>0.1$ & & 8 \\
\hline \multirow{6}{*}{$\begin{array}{l}\text { Irrespective of } \\
\text { conditioning }\end{array}$} & A & Transbond $^{\mathrm{TM}} \mathrm{XT}$ & 13.3 & 8.5 & 1.3 & 10.3 & $<0.001$ & $c, d, e, f$ & \\
\hline & B & Cool-Bond ${ }^{\mathrm{TM}}$ & 11.7 & 7.4 & 1.1 & 9.7 & $>0.1$ & $e, f$ & \\
\hline & C & Ultra Band-Lok & 9.1 & 2.8 & 0.4 & 9.2 & $>0.1$ & $\mathrm{a}, \mathrm{e}$ & \\
\hline & D & Fuji Ortho LC & 8.9 & 5.8 & 0.8 & 7.4 & $>0.1$ & $\mathrm{a}$ & \\
\hline & $\mathrm{E}$ & Tetric $^{\circledR}$ Flow & 6.2 & 2.7 & 0.4 & 6.4 & $>0.1$ & $a, b$ & \\
\hline & $f$ & Light-Bond $^{\mathrm{TM}}$ & 7.5 & 3.5 & 0.5 & 7.3 & $>0.1$ & $a, b$ & \\
\hline
\end{tabular}

Table 3. Shear strengths and standard deviations (SD) of three conditioning methods and comparison by ANOVA. Air abrasion alone showed significantly lower bonding forces than the other two methods.

Tabelle 3. Scherkräfte und Standardabweichungen der drei Konditionierungsmethoden und Vergleich mittels ANOVA. Die alleinige Sandstrahlung wies signifikant niedrigere Haftwerte auf als die beiden anderen Methoden.

\begin{tabular}{lccl}
\hline & Mean [MPa] & SD & Significance (ANOVA, $\mathbf{p} \leq \mathbf{0 . 0 5})$ \\
\hline Etching & 10.8 & 6.1 & Air abrasion \\
Air abrasion & 7.4 & 4.6 & Etching, air abrasion + etching \\
$\begin{array}{l}\text { Air abrasion } \\
+ \text { etching }\end{array}$ & 9.6 & 5 & Air abrasion \\
\hline
\end{tabular}

$\mathrm{MPa}$ ). However, there were no significant differences between the two techniques. Significantly lower adhesive strengths were found for air abrasion alone $(\bar{x}=7.4 \mathrm{MPa})$.

When classified according to the results of the KruskalWallis test, Cool-Bond ${ }^{\mathrm{TM}}$ (etching) and Transbond ${ }^{\mathrm{TM}} \mathrm{XT}$ $(\bar{x}=5,7 \mathrm{MPa})$ und Sandstrahlung kombiniert mit einer Ätzung $(\bar{x}=6,4 \mathrm{MPa})$, sowie für Light-Bond ${ }^{\mathrm{TM}}$ in Kombination mit alleiniger Sandstrahlung $(\bar{x}=4,3 \mathrm{MPa})$ gefunden.

Zwischen den unterschiedlichen Konditionierungstechniken bestanden - unabhängig von den gewählten Adhäsiven - signifikante Unterschiede (Tabelle 3). Die konventionelle Ätzmethode führte zu den höchsten Haftkräften $(\bar{x}=10,8$ $\mathrm{MPa})$, gefolgt von der Kombination Sandstrahlung und Ätzung $(\bar{x}=9,6 \mathrm{MPa})$. Zwischen den oben genannten Konditionierungstechniken bestand kein signifikanter Unterschied, jedoch führte alleinige Sandstrahlung zu signifikant niedrigeren Haftwerten $(\bar{x}=7,4 \mathrm{MPa})$.

Gemäß der Klassifizierung nach Kruskal-Wallis zeigten Cool-Bond ${ }^{\mathrm{TM}}$ (nach Ätzung) und Transbond ${ }^{\mathrm{TM}}$ XT (nach Ätzung) die besten Resultate. Ein signifikanter Unterschied bestand dabei zu allen drei Konditionierungen mit Tetric ${ }^{\circledR}$ Flow und zu Fuji Ortho LC bzw. Light-Bond ${ }^{\mathrm{TM}}$ in Kombination mit Sandstrahlung. An dritter Stelle folgte Fuji Ortho LC bei kombinierter Konditionierungstechnik, an vierter Stelle Transbond $^{\mathrm{TM}}$ XT (nach Sandstrahlung) und an fünfter Stelle 
(etching) ranked in first and second place. A significant difference was found for all Tetric ${ }^{\circledR}$ Flow groups, as well as Fuji Ortho LC and Light-Bond ${ }^{\mathrm{TM}}$, in combination with air abrasion. Ranking third was the glass ionomer Fuji Ortho LC (air abrasion in combination with etching), fourth Transbond $^{\mathrm{TM}}$ XT (air abrasion) and fifth Ultra Band-Lok (etching), which still showed a significant difference to LightBond $^{\mathrm{TM}}$ (air abrasion). No significant differences were found for the remaining adhesives and conditioning techniques.

Overall the adhesive remnant index showed an even distribution of scores (Table 1). $36 \%$ of the probes failed with a score of 1 (adhesive-bracket interface), followed by $34 \%$ with a score of 3 (adhesive-enamel interface) and 30\% with a score of 2 (combination/cohesive fracture).

\section{Discussion}

Enamel conditioning is an essential step in successful bracket bonding. Beside the continuous development of adhesive materials, modifications in enamel conditioning could also enhance adhesive strength. It was the aim of the study to investigate the influence of different conditioning methods (etching, air abrasion, air abrasion + etching) and adhesives on bond strength.

According to ISO 11405/TS, bovine incisors can be used as a substitute for human enamel. Bovine enamel exhibits very similar bonding characteristics to human enamel [1, 22, $35]$ and has often been used as a substitute [16, 32, 34].

The use of brackets was intentionally avoided. Bracket bases have varying bonding surfaces because the bases have different designs with respect to surface area and size. In addition most composites have a low adhesion to metal, which induces a high probability of detachment at the bracket-adhesive interface [10, 24, 31, 33]. This is therefore not ideal when the focus of the study lies in the evaluation of the adhesive-enamel interface. It has been shown that modifications of the experimental setup can lead to variations in shear forces of up to $400 \%$ [10, 24]. We thus chose PMMA cylinders as shear bodies, which allowed the shear procedure to be standardized and chemical adhesion after conditioning with the Rocatec system.

Overall, the use of these PMMA cylinders did not reduce fractures at the interface between adhesive and shear body. The ARI scores of the present study showed an even distribution of fractures. No clear correlation with absolute bond strengths, conditioning technique or adhesive could be found, which is consistent with earlier literature [11, 27, 30] (Tables 1 and 2). The advantage is therefore only the standardization of size, shape, and surface of the PMMA shear bodies.

When considering the overall results of the different adhesives irrespective of the conditioning used, Transbond ${ }^{\mathrm{TM}}$ XT showed the highest bonding strengths, followed by CoolBond $^{\mathrm{TM}}$, the two RMGICs and finally Light-Bond ${ }^{\mathrm{TM}}$ and Tetric $^{\circledR}$ Flow. Higher bonding forces were observed for
Ultra Band-Lok (nach Ätzung), welches sich noch immer signifikant von Light-Bond ${ }^{\mathrm{TM}}$ (nach Sandstrahlung) unterschied. Für die übrigen Adhäsive und Konditionierungsmethoden konnten keine signifikanten Unterschiede gefunden werden.

Die ARI-Werte (Tabelle 1) zeigten eine gleichmäßige Verteilung. 36\% der Proben wiesen einen Score-Wert von 1 (Adhäsiv-Bracket-Grenzfläche) auf, gefolgt von 34\% mit einem Score-Wert von 3 (Adhäsiv-Schmelz-Grenzfläche) und $30 \%$ mit einem Score-Wert von 2 (ähnliche Verteilung der Kompositreste/Kohäsionsfraktur) auf.

\section{Diskussion}

Die Schmelzkonditionierung stellt einen wichtigen Schritt für das erfolgreiche Kleben von Brackets dar. Neben den fortschreitenden Entwicklungen der Adhäsivstoffe können Modifikationen der Schmelzkonditionierung vor dem Bonding die Haftkräfte beeinflussen. Ziel der Studie war es, den Einfluss unterschiedlicher Konditionierungsmethoden (Ätzung, Sandstrahlung, Sandstrahlung und Ätzung) sowie unterschiedlicher Adhäsive auf die Haftkräfte zu untersuchen.

Gemäß der ISO 11405/TS-Norm können bovine Inzisivi als Ersatz für Humanschmelz verwendet werden. Boviner Schmelz weist äußerst ähnliche Hafteigenschaften zu humanem Schmelz auf $[1,22,35]$ und wurde vielfach als Ersatz verwendet $[16,32,34]$.

Die Verwendung von Brackets wurde absichtlich vermieden. Bracketbasen weisen unterschiedliche Oberflächen und Größen auf. Zudem weisen viele Adhäsive ein schlechtes Adhäsionsverhalten zu Metall auf, was eine hohe Wahrscheinlichkeit für einen Klebeverlust am Bracket-Adhäsiv-Übergang darstellt $[10,24,31,33]$. Dies wäre sehr ungünstig gewesen, da der Fokus der Studie auf der Beurteilung der Schmelz-Adhäsiv-Grenzfläche lag. In früheren Studien konnte gezeigt werden, dass Modifikationen des Messaufbaus zu Variationen der Scherkräfte von bis zu 400\% [10,24] führten. Deshalb wurden PMMA-Zylinder als Scherkörper gewählt, welche über das Rocatec-Verfahren eine chemische Adhäsion erlaubten und eine Standardisierung der Schervorgänge ermöglichten.

Insgesamt führte die Verwendung dieser PMMA-Zylinder jedoch nicht zu einer Reduktion der Frakturraten an der Scherkörper-Adhäsiv-Grenzfläche. Die ARI-Werte zeigten eine gleichmäßige Verteilung der Frakturen. Es konnte keine klare Korrelation zu den absoluten Haftkräften, Konditionierungstechniken oder verwendeten Adhäsiven gefunden werden, was in Übereinstimmung mit früherer Literatur steht [11, 27, 30] (Tabellen 1 und 2). Der Vorteil der PMMA-Scherkörper reduzierte sich dadurch auf die verbesserte Standardisierung bezüglich Größe, Form und Oberfläche.

Bei der Betrachtung der verschiedenen Adhäsive unabhängig von der Konditionierungsmethode wies Transbond ${ }^{\mathrm{TM}}$ XT die höchsten Scherkräfte auf gefolgt von Cool-Bond ${ }^{\mathrm{TM}}$, 
Transbond ${ }^{\mathrm{TM}} \mathrm{XT}$ and Cool-Bond ${ }^{\mathrm{TM}}$ compared with RMGICs, which agrees with existing literature [18, 20, 34]. According to the present study the use of RMGICs might be an alternative to conventional composites since standard deviations were smaller, indicating a more uniform bonding behavior. Although the RMGIC Fuji Ortho LC achieved good results in the present study this was not consistent with the literature, which reports varying values, some below $[8,21,35]$ and others above [28] the clinical requirements outlined by Reynolds [25]. It is not clear why Light-Bond ${ }^{\mathrm{TM}}$ did not achieve better shear values. According to earlier studies [2, 37], the smaller amount of fillers in flow-composites such as Tetric ${ }^{\circledR}$ Flow could be responsible for lower shear strengths. However, there is no consensus of opinion in the literature as to whether flowable composites are suitable for orthodontic bonding $[30,33]$.

Taking into consideration that bite forces are 40-120 N and the average bonding area is $16 \mathrm{~mm}^{2}$, a minimum value for successful bonding of 7.5 MPa has been calculated [4]. According to Reynolds [25] a minimum of $6 \mathrm{MPa}$ is recommended. All the tested adhesives displayed acceptable bond strengths. It is also important not to forget maximum bonding limits, as the cohesion of enamel itself lies at 9-11 MPa [3]. Bond strengths far above these values might result in increased enamel fractures during debonding. Maximum force levels are usually evaluated by tensile testing, whereas in the present study shear forces were applied.

Of the three conditioning techniques, significant differences were found between the two techniques involving etching as opposed to air abrasion only (Table 3 ). This is in agreement with previous studies $[5,8,35]$ and is probably due to reduced microroughness of the enamel surface when no etching is involved in the enamel conditioning [35]. Although the combination of air abrasion + etching allows for secure bonding, it must be borne in mind that the combination of both procedures results in a cumulative loss of enamel $[13,35]$ which is not justified clinically since there is no advantage in bond strength. Air abrasion alone, as well as in combination with etching, cannot be recommended.

\section{Conclusion}

Air abrasion alone is insufficient as a conditioning procedure but, in combination with etching, a reasonable bond strength can be achieved. Increased enamel loss due to repeated conditioning must however be considered and for this reason etching alone is preferable.

Resin-modified glass ionomers (RMGICs) generally showed lower shear forces than conventional composites. However, the relative force levels due to the different conditioning methods were similar for RMGIC and composites. The flow-composite Tetric ${ }^{\circledR}$ Flow showed the lowest bond strengths.

The use of PMMA cylinders as shear bodies can only be partially recommended. Although standardization (shape, den beiden RMGICs und Light-Bond ${ }^{\mathrm{TM}}$ sowie Tetric ${ }^{\circledR}$ Flow. Die höheren Haftkräfte für Transbond ${ }^{\mathrm{TM}}$ XT und CoolBond $^{\mathrm{TM}}$ im Gegensatz zu den RMGICs werden in der Literatur bestätigt [18, 20, 34]. Gemäß der vorliegenden Studie könnten die RMGICs eine Alternative zu konventionellen Kompositen darstellen, zumal die Standardabweichung kleiner ausfiel, was auf ein gleichmäßigeres Klebeverhalten hinweist. Der RMGIC Fuji Ortho LC wies in der vorliegenden Studie gute Resultate auf, was in der Literatur nur teilweise bestätigt werden konnte. Einige Autoren wiesen niedrigere Kräfte [8, 21, 35] und andere höhere Kräfte [28] nach als die von Reynolds für die Klinik geforderten Minimalscherkräfte [25]. Unklar blieb, weshalb Light-Bond ${ }^{\mathrm{TM}}$ keine besseren Scherkräfte erreichte. Gemäß früheren Studien [2,37] könnte der niedrigere Füllergehalt von Fließkompositen wie Tetric ${ }^{\circledR}$ Flow für deren niedrige Scherkräfte verantwortlich sein. Ein Konsens zur Eignung von Fließkompositen für das Bracketbonding besteht in der Literatur nicht [30, 33].

Unter Annahme von Kaukräften in der Höhe von 40-120 $\mathrm{N}$ und durchschnittlichen Bondingflächen von $16 \mathrm{~mm}^{2}$ wurde für ein erfolgreiches Bonding ein minimaler Haftwert von 7,5 MPa berechnet [4]. Gemäß Reynolds [25] werden minimale Kräfte von $6 \mathrm{MPa}$ empfohlen. Insofern zeigten alle getesteten Adhäsive akzeptable Haftkräfte. Ebenso wenig sollten die oberen Haftschwellen außer Acht gelassen werden, da die Kohäsion von Schmelz bei 9-11 MPa liegt [3]. Haftkräfte weit über diesem Wert könnten in einer erhöhten Gefahr von Schmelzfrakturen beim Debonding resultieren. Allerdings wurden diese maximalen Kräfte über Zugversuche evaluiert, während in der vorliegenden Studie Scherkräfte appliziert wurden.

Bezüglich der Konditionierungstechniken wurden signifikante Unterschiede zwischen den beiden Methoden Ätzung und alleinige Sandstrahlung gefunden (Tabelle 3). Dies steht im Einklang mit früheren Studien $[5,8,35]$ und ist wahrscheinlich auf die reduzierte Mikrorauheit der Schmelzoberfläche bei unterlassener Ätzung zurückzuführen [35]. Obwohl die Kombination Sandstrahlung + Ätzung ein sicheres Klebeverfahren darstellt, muss beachtet werden, dass die Kombination beider Konditionierungsverfahren in einem kumulativen Schmelzverlust resultiert [13, 35], was klinisch bei gleichen Haftkräften nicht gerechtfertigt ist. Alleinige Sandstrahlung wie auch Sandstrahlung in Kombination mit Ätzung können deshalb nicht empfohlen werden.

\section{Schlussfolgerung}

Sandstrahlung als alleinige Konditionierungstechnik weist ungenügende Haftwerte auf. In Kombination mit Ätzung können angemessene Haftwerte erreicht werden. Jedoch führt eine repetitive Konditionierung zu erhöhtem Schmelzverlust, weshalb die alleinige Ätzung vorzuziehen ist.

Kompositmodifizierte Glasionomerzemente (RMGIC) wiesen insgesamt niedrigere Haftwerte auf als die konventionellen Komposite. Die relativen Haftwerte bezüglich der un- 
bonding surface) is controlled, fractures at the adhesiveshear body interface still occurred in one third of the samples.

\section{Acknowledgement}

We would like to thank the manufacturers who supplied materials for this study. terschiedlichen Konditionierungsmethoden waren für RMGIC und Komposite gleich. Das Fließkomposit Tetric ${ }^{\circledR}$ Flow wies die niedrigsten Haftwerte auf.

Die Verwendung von PMMA-Zylindern als Scherkörper kann nur teilweise empfohlen werden. Obwohl bei diesem Vorgehen die Form und Klebefläche kontrolliert standardisiert werden können, traten dennoch bei einem Drittel der Proben Frakturen an der Adhäsiv-Scherkörper-Grenzfläche auf.

\section{Danksagung}

Wir möchten uns bei allen Industriepartnern für die kostenfreie Materialzusendung für diese Studie bedanken.

\section{References}

1. Bishara SE, VanWald L, Laffoon JF. Effect of altering the type of enamel conditioner on the shear bond strength of a resin-reinforced glass ionomer adhesive. Am J Orthod Dentofacial Orthop 2000;118:288-94.

2. Bishara SE, Gordan VV, VonWald L, Jakobsen JR. Shear bond strength of composite, glass ionomer, and acidic primer adhesive systems. Am J Orthod Dentofacial Orthop 1999;115:24-8.

3. Bowen RL, Rodriguez MS. Tensile strength and modulus of elasticity of tooth structure and several restorative materials. J Am Dent Assoc 1962;64:378-87.

4. Brantley WA, Eliades T. Enamel etching and bond strength. In: Brantley WA, Eliades T, eds. Orthodontic Materials. Scientific and clinical aspects. Stuttgart, New York: Georg Thieme Verlag, 2000;107-20.

5. Brosh T, Strouthou S, Sarne 0. Effects of buccal versus lingual surfaces, enamel conditioning procedures and storage duration on brackets debonding characteristics. J Dent 2005;33:99-105.

6. Buonocore M.G. A simple method of increasing the adhesion of acrylic filling materials to enamel surfaces. J Dent Res 1955;38:849-53.

7. Cacciafesta V, Bosch C, Melsen B. Clinical comparison between a resin-reinforces, self-cured glass ionomer cement and a composite resin for direct bonding of orthodontic brackets. Part 2: Bonding on dry and on enamel soaked with saliva. Clin Orthod Res 1999;2:186-93.

8. Canay S, Kocadereli I, Akça E. The effect of enamel air abrasion on the retention of bonded metallic orthodontic brackets. Am J Orthod Dentofacial Orthop 2000;117:15-9.

9. Carstensen W. Effect of reduction of phosphoric acid concentration on shear bond strength of brackets. Am J Orthod Dentofacial Orthop 1995;108:274-7.

10. Chaconas SJ, Caputo AA, Shi-Lin Niu G. Bond strength of ceramic brackets with various bonding systems. Angle Orthod 1991;61:35-42.

11. Faltermeier A, Behr M, Müssig D. A comparative evaluation of bracket bonding with 1-, 2-, and 3-component adhesive systems. Am J Orthod Dentofacial Orthop 2007;132:144.e1-5.

12. Fuhrmann R, Gutknecht N, Magunski A, et al. Conditioning of enamel with $\mathrm{Nd}: \mathrm{YAG}$ and $\mathrm{CO} 2$ dental laser systems and with phosphoric acid. An in-vitro comparison of the tensile bond strength and the morphology of the enamel surface. J Orofac Orthop 2001;62:375-86.

13. Ireland AJ, Hosein I, Sherriff M. Enamel loss at bond-up, debond and clean-up following the use of a conventional light-cured composite and a resin-modified glass polyalkenoate cement. Eur J Orthod 2005;27:413-9.
14. Jones SP, Gledhill JR, Davies EH. The crystal growth technique - a laboratory evaluation of bond strengths. Eur J Orthod 1999;21: 89-93.

15. Jost-Brinkmann PG, Drost C, Can S. In-vitro study of the adhesive strengths of brackets on metals, ceramic and composite. J Orofac Orthop1996;57:76-87.

16. Keçik D, Cehreli SB, Sar C, Unver B. Effect of acidulated phosphate fluoride and casein phosphopeptide-amorphous calcium phosphate application on shear bond strength of orthodontic brackets. Angle Orthod 2008;78:129-33.

17. Kirovski I, Madzarova S. Tensile bond strength of a light-cured glass ionomer cement when used for bracket bonding under different conditions: an in vitro study. Eur J Orthod 2000;22:719-23.

18. Movahhed $\mathrm{HZ}$, Ogaard B, Syverud M. An in vitro comparison of the shear bond strength of a resin-reinforced glass ionomer cement and a composite adhesive for bonding orthodontic brackets. Eur J Orthod 2005;27:477-83.

19. Newman GV, Newman RA, Sengupta AK. Comparative assessment of light-cured resinmodified glass ionomer and composite resin adhesives: In vitro study of a new adhesive system. Am J Orthod Dentofacial Orthop 2001;119:256-62.

20. Oliveira SR, Rosenbach G, Brunhard IH, et al. A clinical study of glass ionomer cement. Eur J Orthod 2004;26:185-9.

21. Olsen ME, Bishara SE, Damon P, Jakobsen JR. Comparison of shear bond strength and surface structure between conventional acid etching and air-abrasion of human enamel. Am J Orthod Dentofacial Orthop 1997;112:502-6.

22. Oesterle LJ. The use of bovine enamel in bonding studies. Am J Orthod Dentofacial Orthop 1998;113:514-9.

23. Ostby AW, Bishara SE, Laffoon J, Warren JJ. Influence of self-etchant application time on bracket shear bond strength. Angle Orthod 2007;77:885-9.

24. Ostertag AJ, Dhuru VB, Ferguson DJ, Meyer RA. Shear, torsional and tensile bond strengths of ceramic brackets using three adhesive filler concentrations. Am J Orthod Dentofacial Orthop 1991;100:251-8.

25. Reynolds IR. A review of direct orthodontic bonding. $\mathrm{Br} \mathrm{J}$ Orthod 1975:2:171-8.

26. Rix D, Foley T, Mamandras A. Comparison of bond strength of three adhesives: Composite resin, hybrid GIC, and glass-filled GIC. Am J Orthod Dentofacial Orthop 2001;119:36-42. 
27. Scougall Vilchis RJ, Yamamoto S, Kitai N, et al. Shear bond strength of a new fluoride-releasing orthodontic adhesive. Dent Mater $\mathrm{J}$ 2007;26:45-51.

28. Silverman E. A new light cured glass ionomer cement that bonds brackets to teeth without etching in the presence of saliva. Am J Orthod Dentofacial Orthop 1995;108:231-6.

29. Sfondrini MF, Cacciafesta V, Pistorio A, Sfondrini G. Effects of conventional and high-intensity lightcuring on enamel shear bond strength of composite resin and resin-modified glass-ionomer. Am J Orthod Dentofacial Orthop 2001;119:30-5.

30. Tecco S, Traini T, Caputi S, et al. A new one-step dental flowable composite for orthodontic use: An in vitro bond strength study. Angle Orthod 2005;75:574-9.

31. Thind BS, Stirrups DR, Lloyd ChH. A comparison of tungsten-quartzhalogen, plasma arc and light-emitting diode light sources for the polymerization of an orthodontic adhesive. Eur J Orthod 2006;28: 78-82.

32. Thompson MA, Drummond JL, BeGole EA. Bond strength analysis of custom base variables in indirect bonding techniques. Am J Orthod Dentofacial Orthop. 2008;133:9.e15-20.

33. Uysal T, Sari Z, Demir A. Are the flowable composites suitable for orthodontic bracket bonding? Angle Orthod 2004;74:697-702.

34. Valletta R, Prisco D, De Santis R, et al. Evaluation of the debonding strength of orthodontic brackets using three different bonding systems. Eur J Orthod. 2007;29:571-7.
35. Van Waveren Hogervorst WL, Feilzer AJ, Prahl-Andersen B. The airabrasion technique versus the conventional acid-etching technique: A quantification of surface enamel loss and a comparison of shear bond strength. Am J Orthod Dentofacial Orthop 2000;117:20-6.

36. Wickwire NA, Rentz D. Enamel pre-treatment: A critical variable in direct bonding systems. Am J Orthod 1973;64:499-512.

37. Willems G, Carels CE, Verbeke G. In vitro peel/shear bond strength of orthodontic adhesives. J Dent 1997;25:263-70.

\section{Correspondence Address}

Dr. Dr. Lorenz Brauchli

Clinic of Orthodontics and Pedodontics University of Basel

Hebelstr. 3

4056 Basel

Switzerland

Phone: (+41/61) 26726-41, Fax -57

e-mail: lorenz.brauchli@unibas.ch 